\title{
HEAT CAPACITY OF THE TIN NEUTRON-RICH ISOTOPES, INCLUDING QUANTAL AND STATISTICAL FLUCTUATIONS
}

\author{
H. Kadi* \\ Center for Research in Astronomy and Astrophysics and Geophysics (CRAAG), \\ and Laboratoire de Physique Théorique, Faculté de Physique,USTHB \\ BP 32, El Alia, 16111 Bab Ezzouar, Algiers, Algeria \\ E-mail: h.kadiecraag.dz
}

N. Benhamouda

Laboratoire de Physique Théorique, Faculté de Physique,USTHB

BP 32, El Alia, 16111 Bab Ezzouar, Algiers, Algeria

\section{A. Haddouche}

Laboratoire de Physique Théorique, Faculté de Physique,USTHB

BP 32, El Alia, 16111 Bab Ezzouar, Algiers, Algeria,

and Faculté des Sciences et de la Technologie, Université Dr. Yahia Fares de Médéa, Quartier

Ain D’heb, 26000 Médéa, Algeria

\begin{abstract}
At finite temperature, very limited information exists on nuclear level density and thermodynamic properties for such nuclei. So, one proposes in the present work to study the phenomenon of pairing phase transition, by evaluating the heat capacity, in the case of Tin isotopes. So, as a first step, our study will include the ordinary $\mathrm{Sn}$ isotopes such as $116 \leq A \leq 119$, it will then be extended to the neutron-rich nuclei such as $126 \leq A \leq 129$. Theoretically, we use the modified Lipkin-Nogami method (MLN) to eliminate the quantal and statistical fluctuations inherent in the FTBCS approach. The obtained results are compared to the conventional FTBCS results and to the MBCS predictions as well as to the experimental data when available. The inclusion of quantal and statistical fluctuations induces S-shape in the heat capacity curves, which is in a good agreement with experimental data.
\end{abstract}

XIII Nuclei in the Cosmos

7-11 July, 2014

Debrecen, Hungary

\footnotetext{
* Speaker.
} 


\section{Introduction}

Properties of the nuclei at extreme conditions are important in various astrophysical scenarios such as in late stage of a supernova collapse and explosion. During this phenomenon, neutron-rich hot heavy nuclei can be produced. So, the study of properties of these exotic nuclei is essential for understanding processes in nuclear astrophysics. The fact that these nuclei present an important $\mathrm{N} / \mathrm{Z}$ ratio induces a fundamental change either in the nuclear density or in the effective interactions. At finite temperature, very limited information exists on nuclear level density and thermodynamic properties particularly for such nuclei. In the present paper, one proposes to study the phenomenon of pairing phase transition, by studying the heat capacity, in the case of Sn neutron-rich isotopes such as $66 \leq N \leq 79$ including even-even and even-odd isotopes.

The study of pairing phase transitions in the hot nucleus presents actually a real challenge. This phenomenon was well described by the FTBCS approach [四]. However, this approach neglect of the quantal and the statistical fluctuations in the mean field approximations. The quantal fluctuations arise from the fact that the BCS state is not eigen state of the particles number operator. The elimination of this kind of fluctuations can be done using the particle number projection techniques. The Lipkin-Nogami (LN) method is simple and numerically powerful to use and has thus become the standard method for restoring the particle number symmetry.

On the other hand, the BCS theory at finite temperature (FTBCS) violates the unitary relation of the particle-density matrix, inducing statistical fluctuations, making the FTBCS results invalid at high temperatures. In recent years, many methods have been introduced to treat statistical fluctuations, principally to explain the persistence of the pairing beyond the critical temperature provided by the FTBCS method, and "S" shape in heat capacity was detected experimentally [四]-[四]. So, in the present work, one proposes to use the modified Lipkin-Nogami method (MLN) [[], 目] to eliminate the quantal and statistical fluctuations inherent in the FTBCS approach. The method is applied to evaluation of the heat capacity. The obtained results are compared to MBCS [U] and FTBCS predictions as well as to the experimental data when available. The paper is organized as follows: the formalism is presented in section 2. Numerical results are discussed in section 3. Main conclusions are summarized in the same section.

\section{Formalism}

In the second quantization, the Hamiltonian that describes a system of $N=2 P$ particles, where $\mathrm{P}$ is pairs of paired particles, is given by:

$$
\hat{H}=\sum_{\nu>0} \varepsilon_{v}\left(a_{v}^{\dagger} a_{v}+a_{\tilde{v}}^{\dagger} a_{\tilde{v}}\right)-G \sum_{\nu \mu>0} a_{v}^{\dagger} a_{\tilde{v}}^{\dagger} a_{\tilde{\mu}} a_{\mu},
$$

where $a_{v}^{\dagger}$ and $a_{v}$ respectively represent the creation and annihilation operators of the state $|v\rangle$, of energy $\varepsilon_{v}$; and $a_{\tilde{v}}^{\dagger}$ and $a_{\tilde{v}}$ those of the state $|\tilde{v}\rangle$, which is the time reverse of $|v\rangle$ and has the same energy. $\mathrm{G}$ is the pairing strength and assumed to be constant.

Let us recall, that in the case of the FTBCS approach, the use of the canonical Bogoliubov transformation from the particle operators $a_{v}^{\dagger}$ and $a_{v}$ to the quasi-particle ones $\alpha_{v}^{\dagger}$ and $\alpha_{v}$ :

$$
a_{v}^{\dagger}=u_{v} \alpha_{v}^{\dagger}+v_{v} \alpha_{\tilde{v}}
$$




$$
a_{\tilde{v}}=u_{v} \alpha_{\tilde{v}}-v_{v} \alpha_{v}^{\dagger}
$$

allows one to obtain the FTBCS gap equations in the form [四:

$$
\begin{aligned}
& \Delta=G \sum_{v \succ 0} u_{v} v_{v}\left(1-2 \eta_{v}\right) \\
& N=2 \sum_{v \succ 0}\left[v_{v}^{2}+\left(1-2 v_{v}^{2}\right) \eta_{v}\right]
\end{aligned}
$$

where $\eta_{v}$ is the quasi-particle occupation number given by the Fermi-Dirac distribution $\eta_{v}=\frac{1}{1+\exp \left(\beta E_{v}\right)}$ and $E_{v}=\sqrt{\left(\varepsilon_{v}-\lambda\right)^{2}+\Delta^{2}}$

At finite temperature as it has been shown in ref [[]] , one has:

$$
\operatorname{Tr}\left(R-R^{2}\right)=2 \sum_{v \succ 0} \eta_{v}\left(1-\eta_{v}\right) \equiv 2(\delta N)^{2} \neq 0
$$

where $(\delta N)$ is a quasi-particle-number fluctuation. As a consequence, the FTBCS theory violates the unitary relation for the generalized particle-density matrix $\mathrm{R}$, which requires $R^{2}=R$.

To overcome this defect, a secondary Bogoliubov transformation was introduced:

$$
\begin{aligned}
& \bar{\alpha}_{v}^{\dagger}=\sqrt{1-\eta_{v}} \alpha_{v}^{\dagger}+\sqrt{\eta_{v}} \alpha_{\tilde{v}} \\
& \bar{\alpha}_{\tilde{v}}=\sqrt{1-\eta_{v}} \alpha_{\tilde{v}}-\sqrt{\eta_{v}} \alpha_{v}^{\dagger}
\end{aligned}
$$

this transformation connects between the usual quasiparticle (QP) operators $\alpha_{v}^{\dagger}$ and $\alpha_{v}$ and the modified quasiparticle operators (MQP) $\bar{\alpha}_{v}^{\dagger}$ and $\bar{\alpha}_{v}$. Using the transformations (‥2) and (‥6), one has:

$$
\begin{aligned}
& a_{v}^{\dagger}=\bar{u}_{v} \bar{\alpha}_{v}^{\dagger}+\bar{v}_{v} \bar{\alpha}_{\tilde{v}} \\
& a_{\tilde{v}}=\bar{u}_{v} \bar{\alpha}_{\tilde{v}}-\bar{v}_{v} \bar{\alpha}_{v}^{\dagger}
\end{aligned}
$$

where the variational parameters $\bar{u}_{v}$ and $\bar{v}_{v}$ read:

$$
\begin{aligned}
& \bar{u}_{v}=\sqrt{1-\eta_{v}} u_{v}+\sqrt{\eta_{v}} v_{v} \\
& \bar{v}_{v}=\sqrt{1-\eta_{v}} v_{v}-\sqrt{\eta_{v}} u_{v}
\end{aligned}
$$

that allows to take into account the statistical fluctuations, and define a modified gap equations:

$$
\begin{aligned}
& \bar{\Delta}=G \sum_{v \succ 0}\left\langle a_{\tilde{v}} a_{v}\right\rangle=G \sum_{v \succ 0}\left(u_{v} v_{v}\left(1-2 \eta_{v}\right)-\left(u_{v}^{2}-v_{v}^{2}\right) \delta \eta_{v}\right) \\
& N=2 \sum_{v \succ 0}\left\langle a_{v}^{\dagger} a_{v}\right\rangle=2 \sum_{v \succ 0}\left(v_{v}^{2}+\left(u_{v}^{2}-v_{v}^{2}\right) \eta_{v}-2 u_{v} v_{v} \delta \eta_{v}\right)
\end{aligned}
$$

where $\delta \eta_{v}=\sqrt{\eta_{v}\left(1-\eta_{v}\right)}$ is a quasiparticle fluctuation number on the $v^{\text {th }}$ orbital.

It is worth noticing here that the modified quasiparticle energy $\bar{E}_{v}=\sqrt{\left(\varepsilon_{v}-\bar{\lambda}\right)^{2}+\bar{\Delta}^{2}}$ replaces the quasiparticle energy $E_{v}$ in the expression of the quasiparticle-occupation number $\eta_{v}$.

The Modified BCS approach (MBCS) allows one to establish the gap equations in a simple way. However, it neglects the fluctuations of the particle number. This defect may be eliminated using the 
Modified Lipkin-Nogami method (MLN). That consist on introduction of the $u_{v}$ and $v_{v}$ coefficients normalized by the standard Lipkin Nogami, and inject them in the MBCS gap equations, such as:

$$
\left.\begin{array}{c}
u_{v}^{2} \\
v_{v}^{2}
\end{array}\right\}=\frac{1}{2}\left\{1 \pm \frac{\varepsilon_{v}-\lambda+\left(4 \lambda_{2}-G\right) v_{v}^{2}}{\sqrt{\left(\varepsilon_{v}-\lambda-G v_{v}^{2}+4 \lambda_{2} v_{v}^{2}\right)^{2}+\bar{\Delta}^{2}}}\right\}
$$

The usual LN $\lambda_{2}$ coefficient then becomes:

$$
\lambda_{2}=\frac{G}{4} \frac{\sum_{v \succ 0} \bar{u}_{v}^{3} \bar{v}_{v} \sum_{v \succ 0} \bar{u}_{v} \bar{v}_{v}^{3}-\sum_{v>0} \bar{u}_{v}^{4} \bar{v}_{v}^{4}}{\left(\sum_{v \succ 0} \bar{u}_{v}^{2} \bar{v}_{v}^{2}\right)^{2}-\sum_{v \succ 0} \bar{u}_{v}^{4} \bar{v}_{v}^{4}}
$$

The quasi-particle occupation number is defined by $\eta_{v}=\frac{1}{1+\exp \left(\beta \bar{E}_{v}\right)}$ where the modified quasiparticle energy $\bar{E}_{v}=\sqrt{\left(\varepsilon_{v}-\lambda-G \bar{v}_{v}^{2}+4 \lambda_{2} \bar{v}_{v}^{2}\right)^{2}+\bar{\Delta}^{2}}$

\subsection{Heat capacity}

The heat capacity study informs on the nature and trends in the system, and shows any phase transitions that can occur with increasing the temperature.

The heat capacity of even-even isotopes is defined as $C=\frac{d E_{M L N}}{d T}$, where $E_{M N L}$ is the Modified Lipkin-Nogami internal energy, it is given by:

$$
E_{M L N}=2 \sum_{v \succ 0}\left(\varepsilon_{v}-G \bar{v}_{v}^{2}+4 \lambda_{2} \bar{v}_{v}^{2}\right) \bar{v}_{v}^{2}-\frac{\bar{\Delta}^{2}}{G}-4 \lambda_{2} \sum_{v \succ 0} \bar{u}_{v}^{2} \bar{v}_{v}^{2}
$$

Concerning the even-odd isotopes, one uses the entropy definition [Q]:

$$
S_{\text {even-odd }}=S_{\text {even-even }} \frac{1+n_{q p}}{n_{q p}}
$$

where $n_{q p}=2 \sum_{v \succ 0} \eta_{v}$ and $S_{\text {even-even }}=2 \sum_{v \succ 0}\left(\ln \left(1+\exp \left(-\beta E_{v}\right)\right)+\frac{\beta E_{v}}{1+\exp \left(-\beta E_{v}\right)}\right)$

The heat capacity is thus deduced by: $C=T \frac{S_{\text {even-odd }}}{d T}$

\section{Numerical results and discussion}

The MLN method was applied to the calculation of heat capacity of the Tin ordinary isotopes. We used the single-particles and eigen-states of a deformed Woods-Saxon mean-field. We considered a maximum number of shells $N_{\max }=12$. The study will include the ordinary isotopes, it will then be extended to the neutron-rich nuclei. In order to compare the theoretical predictions to the experimental data, we used the values of the experimental level density $\rho\left(E_{n}\right)$ to determine the partition function in the canonical ensemble, using the following expression [ए]]:

$$
Z(T)=\sum_{n \succ 0} \rho\left(E_{n}\right) \exp \left(-\frac{E_{n}}{T}\right)
$$

where $E_{n}$ is the excitation energy. The sum must be infinite, but as the level density is obtained for maximum excitation energy of $9 \mathrm{MeV}$. For energy values greater than $9 \mathrm{MeV}$, the level density is 

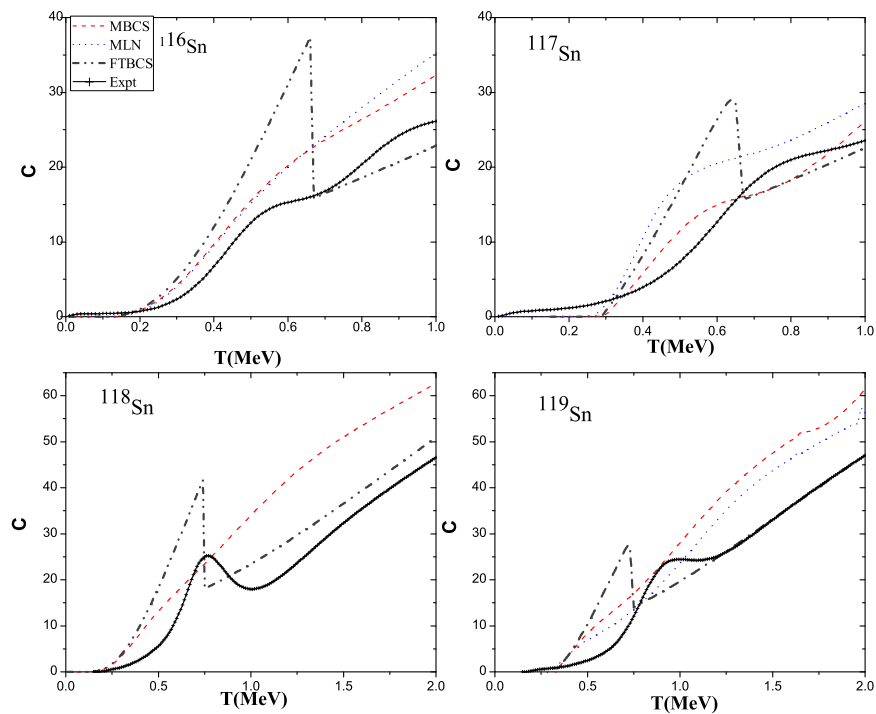

Figure 1: Variation of the heat capacity as a function of temperature in the case of Tin ordinary isotopes $116 \leq A \leq 119$

extrapolated using the formula of "Back-Shifted Fermi gas" model. The calculation of total energy and heat capacity is done by the following relations:

$$
\begin{aligned}
E(T) & =\frac{\sum_{n \succ 0} E_{n} \rho\left(E_{n}\right) \exp \left(-\frac{E_{n}}{T}\right)}{Z(T)} \\
C & ==\frac{d E(T)}{d T}
\end{aligned}
$$

In a first step, we have evaluated the heat capacity as a function of temperature in the case of ordinary nuclei such as $116 \leq A \leq 119$. The MLN results are reported in figure $\mathbb{W}$. We have also plotted in the same figure, the experimental data as well as the theoretical predictions of FTBCS and MBCS approaches. One notices:

- The FTBCS results present peaks signatures of sharp phase transition.

- The heat capacity curves calculated within MBCS and MLN methods present S shape similar to experimental calculation. It appears thus the importance of quantal and statistical fluctuations.

- At high temperatures the FTBCS method reproduces better experimental values.

- We also note that the MLN results are in better agreement with the experimental data as the neutron number increases. That means that quantal fluctuations depend on the neutron number.

As a second step, one reports in figure $\square$ the predictions of heat capacity for neutron-rich isotopes such as $126 \leq A \leq 129$. one may note from the figure: 

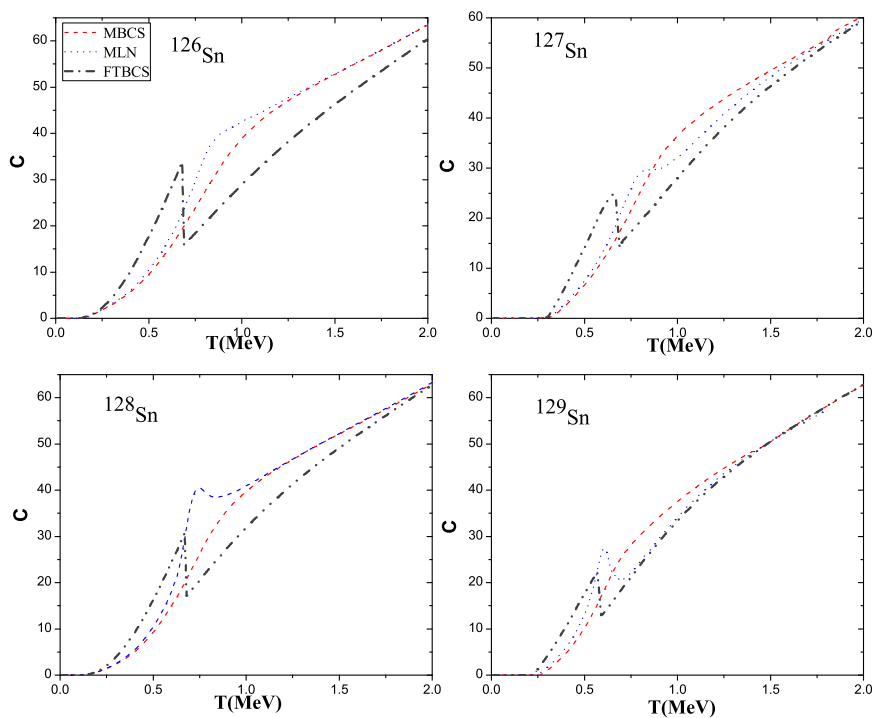

Figure 2: Variation of the heat capacity as a function of temperature in the case of Tin neutron-rich isotopes $126 \leq A \leq 129$

- A sharp phase transition appears in the case of FTBCS method.

- A S shape phase transition within MBCS and MLN methods. The S shape clearly appears when MLN method is used. Moreover, it is pronounced when neutron number increases specially near the neutron shell closure $\mathrm{N}=82$. In this region, the quantal fluctuations play an important role.

- The three methods converge at high temperatures.

\section{References}

[1] L. G. Moretto, Phys. Lett. B 40, 1 (1972).

[2] U. Agvaanluvsan, Phys. Rev. C 79, 014320 (2009).

[3] K. Toft et al, Phys. Rev. C 81, 064311 (2010).

[4] H. K. Toft et al, Phys. Rev. C 83, 044320 (2011).

[5] N. Dinh Dang, Phys. Rev.C 76, 064320 (2007).

[6] H. Kadi, et al, Int. J. Mod. Phys. E 22, 1350034 (2013).

[7] N. Dinh Dang, and A.Arima, Phys.Rev. C 67, 014304(2003).

[8] A.L. Goodman, Nucl.Phys. A 352, 30 (1981).

[9] M. Guttormsen et al, Phys. Rev. C 62, 024306 (2000).

[10] A. Schiller et al, Phys. Rev.C 63, 021306(R) (2001). 